\begin{abstract}
1
\section{UN MODELO PARA INTEGRAR \\ LENGUAJE Y CONOCIMIENTO DEL MUNDO: APRENDER A TRAVÉS DE LA LENGUA}

A MODEL FOR INTEGRATING LANGUAGE AND KNOWLEDGE

OF THE WORLD: LEARNING THROUGH LANGUAGE
\end{abstract}

Carmen Marimón Llorca

marimon@ua.es /

Universidad de Alicante

España. 
RESUMEN

El objetivo de este trabajo es mostrar cómo a través de la práctica de determinadas estrategias discursivas es posible que el/la estudiante de español como lengua extranjera comprenda un texto complejo como una totalidad, se acerque a los significados esenciales y se despierte en él el espíritu crítico. A partir del nuevo diseño de enseñanza/aprendizaje que suponen el MCER y el PCIC por lo que se refiere a la integración de los conceptos de interculturalidad y conocimiento social, se muestra la presencia, en el PCIC, de conceptos propios de la LSF y su desaprovechamiento a la hora de plantear un aprendizaje de la lengua socialmente orientado. La aplicación se realiza sobre el concepto de ciudadanía por lo que se explica en qué consisten los contenidos de la materia Educación para la ciudadanía (por lo que se refiere al Estado español) y se justifica su pertinencia para enseñar competencias lingüísticas e interculturales. Finalmente, se ejemplifica el planteamiento teórico con un análisis de tres fragmentos de libros de texto a partir de las metafunciones de Halliday. Se concluye con una reflexión sobre las posibilidades reales de vincular lengua y sociedad y de, tal y como se propone en el funcionalismo, «aprender a través de la lengua» también una lengua extranjera.

\section{PALABRAS CLAVE}

$>$ lingüística sistémico funcional

$>$ español como lengua extranjera

$>$ análisis del discurso

$>$ gramática

$>$ interculturalidad 


\section{ABSTRACT}

The objective of this work is to show how through the practice of certain discursive strategies it is possible for the student of Spanish as a foreign language to understand a complex text as a whole, get closer to the essential meanings and awaken the spirit critical. From the new teaching / learning design that the MCER and the $P C I C$ suppose with regard to the integration of the concepts of interculturality and social knowledge, the presence, in the PCIC, of the concepts of the LSF and its waste when proposing a socially oriented learning of the language. The application is carried out on the concept of citizenship, so it is explained what the contents of the Education for Citizenship course consist of (as regards the Spanish State) and its relevance to teach linguistic and intercultural competences is justified. Finally, the theoretical approach is exemplified with an analysis of three textbook fragments based on Halliday's metafunctions. We conclude with a reflection on the real possibilities of linking language and society and of, as proposed in functionalism, «learning through language» also a foreign language.

\section{KEYWORDS}

$>$ systemic functional linguistics

$>$ Spanish as a foreign language

$>$ discourse analysis

$>$ grammar

$>$ interculturality 


\section{INTRODUCCIÓN: LENGUA, INTERCULTURALIDAD Y CULTURA CIUDADANA}

Una de las aportaciones más importantes tanto del MCER como del PCIC es el interés planificado que muestran por desarrollar en el estudiante de L2/LE competencias relacionadas con habilidades y actitudes interculturales, así como con saberes y comportamientos socioculturales. Es en el MCER donde aparece, por primera vez, el concepto de competencia intercultural como parte integrante del proceso de aprendizaje. En él, el desarrollo de la «consciencia intercultural» forma parte, por un lado, de los «saberes» declarativos que el estudiante debe desarrollar. Pero no se trata únicamente de la adquisición de conocimiento objetivos, de datos sobre la cultura objeto de estudio, sino, como se señala en el texto, es también «una toma de conciencia del modo en que aparece la comunidad desde la perspectiva de los demás, a menudo en forma de estereotipos nacionales». Esto implica entender la vida social y las relaciones que se dan en una comunidad; por eso, el desarrollo de la interculturalidad es también considerada parte de los conocimientos instrumentales, del saber hacer del estudiante. Pero, además, y como se ha señalado en publicaciones recientes, el conocimiento intercultural está estrechamente relacionado con el «saber ser», es decir, con la competencia existencial, en la medida en que esta tiene que ver con las actitudes, las motivaciones, los valores, las creencias, los estilos cognitivos y los tipos de personalidad que contribuyen a su identidad personal.

EI PCIC por su parte, muestra un claro interés por desarrollar en el estudiante de español competencias relacionadas con las habilidades y actitudes interculturales, así como con los saberes y comportamientos socioculturales; de hecho, los términos con los que en el PCIC se definen «agente social» o "hablante intercultural» están muy cercanos a conceptos como el de «ciudadanía» con lo que converge con la implantación en muchos países del ámbito hispánico de materias curriculares - como en España, Educación para la ciudadanía- con las que se ha querido avanzar en las competencias sociales, éticas y críticas que ayudarán al estudiante a ser un adulto responsable. En España se da el caso de que, desde hace varias décadas, en las aulas de educación secundaria conviven adolescentes de distintas nacionalidades y lenguas, muchos de los cuales tienen que simultanear los procesos de integración cultural, aprendizaje de la lengua y avance en el currículum escolar. Para ellos, con un dominio inseguro del español y 
unos referentes culturales en ocasiones muy diferentes, los textos de una materia como esta, que incluyen conceptos complejos, como derechos, minoría, clase social, no sólo está probablemente muy lejos de su nivel de lengua, sino, lo que es más importante, de sus posibilidades reales de darles sentido.

Es en esta relación entre las ideas de convivencia y diálogo entre culturas, por un lado, y el hecho del aprendizaje de una nueva lengua — L2 - por parte de estudiantes jóvenes por otro, lo que nos ha hecho plantearnos la necesidad de establecer una relación entre lengua e interculturalidad. Nuestra pregunta de investigación tiene que ver con la posibilidad de conectar los contenidos lingüísticos y los interculturales en un currículum de español L2. Creemos que la respuesta debe ser afirmativa y lo creemos porque ni unos conocimientos -los lingüísticos- ni los otros, -los interculturales - son, como hemos visto que ya señala el MCER sólo «saberes», sino que son, sobre todo, habilidades, destrezas, comportamientos indisociables de las prácticas sociales que el hablante realiza cotidianamente; y esas prácticas, en una gran medida, son, sobre todo, prácticas verbales. El objetivo de este trabajo es mostrar cómo a través de la práctica de determinadas estrategias discursivas se puede facilitar que el estudiante comprenda un texto complejo como una totalidad, se acerque a los significados esenciales y despierte en él el espíritu crítico. Asumimos que, a través del análisis y comprensión de las herramientas lingüísticas comunicativas en una lengua, sea esta L2 o L1, es decir, de sus discursos, el estudiante puede y debe desarrollar el espíritu crítico y la conciencia social que le darán valor como ciudadano.

Con este fin, en primer lugar, se establece el marco teórico por lo que se refiere a la concepción de la lengua que manejamos al hilo del análisis de lo que se dice al respecto en el $P C I C$. Se hace hincapié en la presencia de conceptos propios de la LSF y en su desaprovechamiento a la hora de plantear un aprendizaje de la lengua socialmente orientado. A continuación, se profundiza en el concepto de ciudadanía, particularmente por lo que se refiere al Estado español, se explica en qué consisten los contenidos de esta asignatura y se justifica su pertinencia para enseñar competencias lingüísticas e interculturales. Finalmente, se ejemplifica el planteamiento teórico con un análisis de tres fragmentos de libros de texto de EPC a partir de las metafunciones de Halliday. Se concluye con una reflexión sobre las posibilidades reales de vincular lengua y sociedad y de, tal y como se propone en el funcionalismo, «aprender a través de la lengua» también una lengua extranjera. 


\section{LENGUA Y APRENDIZAJE INTERCULTURAL EN EL PCIC}

En la «Introducción general» del $P C I C$ se explica que el enfoque adoptado en la estructuración de los niveles de referencia para el español se hace desde el análisis de la lengua basado en la comunicación, es decir, que, para el análisis de la lengua, se va a tener en cuenta la participación de los actores en el evento comunicativo. Este propósito se concreta en la doble perspectiva conceptual que organiza y justifica todo el plan de la obra al considerar, respectivamente, al alumno y a la lengua como sujeto y objeto del aprendizaje. Así, si la materia objeto de estudio es la lengua española, el español, el verdadero protagonista del aprendizaje será el aprendiente, el individuo que como potencial usuario de la lengua se acerca a esta para ejercitarla en la comunicación efectiva, planificadamente, en situaciones contextualmente determinadas, sean esta del tipo que sean. Desde esta perspectiva sujetual, el aprendiente no es visto de forma plana como un participante pasivo (valga el oxímoron), ni siquiera sólo como alguien con necesidades lingüístico-comunicativas, sino que se consideran en él «tres dimensiones complementarias e independientes», cuyo desarrollo es paralelo y debería ser inseparable del proceso de aprendizaje de las herramientas lingüísticas necesarias para la comunicación. Se trata de las dimensiones del alumno como agente social, como hablante intercultural y como aprendiente autónomo. Aunque todo el mundo parece saber de memoria esta tríada competencial, en mi opinión no se ha reflexionado lo suficiente sobre la manera en que estas dimensiones pueden trabajarse $y$, sobre todo, creo que no se ha hecho intentado relacionar el desarrollo de estas habilidades lingüístico-sociales con la propia dinámica de enseñanza/aprendizaje de la lengua. En general, en los libros de texto la cuestión se solventa con la alusión a contenidos culturales, ciertamente cada vez más y mejor integrados - hábitos, costumbres, tópicos, etc.-y, en niveles avanzados, con prácticas sobre el uso adecuado de distintos registros a través de muestras reales de lengua. Creo, sin embargo, que se puede dar un paso más e intentar que el desarrollo de estas dimensiones sociales y culturales en el alumno (dejamos en este caso para otra ocasión la vertiente de aprendiente autónomo) venga de la mano de la práctica del aprendizaje de la lengua. Como vamos a ver, el PCIC se acerca a esta posibilidad, aunque no llega a materializarla metodológicamente. 
Entendida como objeto de aprendizaje, la lengua en el PCIC se observa desde las perspectivas psicológicas y lingüísticas más avanzadas —cognitivismo, Pragmática, Análisis del discurso-, en definitiva, las que representan y construyen el llamado modelo o paradigma funcional en lingüística. Para justificar teóricamente la orientación competencial y funcional sobre la lengua que se propone, el PCIC recurre a los fundamentos mismos del funcionalismo lingüístico: Hymes y Halliday. Así, para la organización de la competencia verbal se parte del concepto de competencia comunicativa de Hymes ${ }^{1}$, para quien esta consta de una competencia gramatical, una competencia estratégica y una competencia textual ${ }^{2}$. Se hace notar entonces que la propuesta de Halliday (1982) sobre las macrofunciones del lenguaje resulta idónea y encaja muy ajustadamente con el modelo competencial de Hymes pues distingue una función ideacional, que García Nieto (p. 36) hace coincidir con la competencia gramatical debido a que esta se realiza a través de índices como la transitividad; una función interpersonal, que mediante las categorías de la enunciación enlaza con la competencia estratégica; y una función textual que tiene que ver con los rasgos de la textualidad. Pero el PCIC no va más allá del establecimiento de estas conexiones lo que se hace evidente cuando vemos que el planteamiento trifuncional de Halliday se utiliza sólo para justificar un enfoque de la lengua que atiende a cuatro tipos de competencias, las tres que se derivan de Hymes y las funciones de Halliday que son la léxico-gramatical, la interpersonal y la textual, a la que se añade una competencia enciclopédica que, contraviniendo las bases de la teoría funcional, introduce el conocimiento cultural o del mundo como algo diferente a la propia lengua, desligado de las funciones del lenguaje, tal y como, en efecto, sucede en la mayoría de los manuales. Finalmente, de estas competencias se hacen derivar los cinco componentes de la competencia verbal: gramatical, pragmático-discursivo (funciones, estructuras pragmáticas, géneros discursivos y productos textuales), nocional, cultural y de aprendizaje.

\footnotetext{
${ }^{1}$ Aunque todos deudores del modelo de Hymes, en los últimos años se han desarrollados modelos competenciales pensados expresamente para la enseñanza de segundas lenguas. Es el caso, por ejemplo, de la propuesta de Canale y Swain $(1980,1983)$ que distingue cuatro competencias: gramatical, sociolingüística, estratégica y discursiva. Y, más recientemente de la de Bachman (1990) que diferencia una competencia organizativa, dividida a su vez en gramatical y textual; y una competencia pragmática, con la correspondientes subcompetencias ilocutiva y sociolingüística. Ver Cenoz ( )

2 Este trabajo es una reelaboración y una revisión de trabajos anteriores: Marimón, 2009 y 2016).
} 
Lo que hemos querido mostrar con este recorrido es que el $P C I C$, aunque recurre inicialmente al modelo de Halliday, pasa de largo sobre el potencial didáctico e integrador de los saberes sociales y lingüísticos que ésta encierra. Desde su concepción del lenguaje como semiótica social, Halliday nunca concibió la lengua como algo distinto del mundo, sino que, para él y su escuela, la Lingüística sistémico-funcional (LSF), la naturaleza del sistema lingüístico se explica precisa y únicamente en relación con las metafunciones o significados que los hablantes quieren transmitir y, estos, a su vez, con las condiciones que enmarcan dichos intercambios, es decir, con los contextos de uso. Se establece así un puente coherente entre los indicadores léxico-gramaticales y semánticos, las funciones que realiza el lenguaje como hecho sociocomunicativo y las parcelas del contexto que resultan relevantes en los distintos actos de comunicación.

En cuanto al sujeto del aprendizaje, hay que señalar que a la concepción competencial y funcional de la lengua le corresponde un sujeto cuyo aprendizaje está basado en la acción. Por eso en el PCIC se le concibe como un agente social, que conoce el sistema de la lengua y sabe desenvolverse en situaciones de interacción; un hablante intercultural que reconoce y respeta una nueva cultura con sus valores y creencias y sabe relacionarse con ella desde los suyos; y un aprendiente autónomo, que controla su proceso de aprendizaje y adquiere un papel activo sobre él. Si nos centramos en las dos primeras dimensiones, podemos concluir que lo que se espera en el $P C I C$ de un estudiante de español es que entienda que no va sólo a «informarse» sobre un nuevo sistema lingüístico, sino que, además, va a formarse como individuo capaz de identificar situaciones sociales, capaz de manejar recursos para poder actuar social y verbalmente de manera adecuada en distintos escenarios, que deberá desarrollar una imagen de sí mismo y de los otros respetuosa con la complejidad de la sociedad actual y reconocer críticamente los sistemas de valores y creencias en su gran diversidad, dentro de su propio referente sociocultural y en relación con el que está adquiriendo. Es, sin duda, un programa muy ambicioso que supera con mucho la concepción clásica de un programa de lenguas para sumergirse de lleno en una concepción activa y comprometida del aprendizaje.

Esta concepción social de la lengua y el aprendizaje resulta, en mi opinión, no sólo muy atractiva, sino que puede encajar muy bien con las necesidades de conocimiento instrumentales y socio-culturales que necesitan los alumnos no nativos en sus años de escolarización en el sistema educativo español. 


\section{EL CONCEPTO DE "CIUDADANÍA» Y LOS OBJETIVOS DE LA ENSEÑANZA: INFORMAR Y FORMAR}

Con la implantación de la materia Educación para la Ciudadanía en el ciclo obligatorio de enseñanza secundaria, se quiso avanzar en la formación de competencias sociales, éticas y críticas que ayudarán al estudiante a ser en un futuro cercano un adulto responsable. Aunque en España el término aplicado a la enseñanza es relativamente nuevo, hace años que en la escuela Latinoamericana la preocupación porque los estudiantes se formen como ciudadanos es una constante en congresos de docentes y asociaciones de padres y madres. Quizá los enormes problemas de desigualdad social y desarraigo familiar que recorren el continente tengan que ver en el especial interés que los docentes ponen en este aspecto de la educación, pues ven en la educación misma y en la formación de ciudadanos una puerta hacia el futuro de sus países o regiones. Así, Griselda Gabalachis (2009: p. 1) plantea la educación como «esa herramienta necesaria para llevar adelante un modelo de desarrollo sustentable, que tiene que ver con entender la Educación como creadora de Ciudadanía, de inclusión y de movilidad social». Desde este punto de vista, educar para la ciudadanía es algo más que impartir una nueva materia curricular, se trata de orientar al estudiante en su propio crecimiento como individuo crítico, activo y participativo. A este respecto señala Silvia Coicaud (2009: p. 1):

En este sentido, la enseñanza para la ciudadanía puede constituirse en un proyecto pedagógico innovador, asumiendo especial relevancia el concepto de enseñanza como práctica humana que compromete moral socialmente a quienes la realizan, a partir de la formación de los jóvenes como sujetos de derecho que contribuirán al mejoramiento de la sociedad en la que vivimos.

A propósito de esta concepción formativa y social del proceso educativo, Silvia Coicaud (2009: p. 6) propone abordar la ciudadanía como una práctica contextualizada que permite a los sujetos decodificar de manera reflexiva los lenguajes, ritos y símbolos propios de un espacio social.

En Europa y en España (Souto, 2009) la preocupación por este aspecto esencial de la educación de los jóvenes no es menor y, de hecho, está presente tanto en las recomendaciones del Consejo de Europa del año 2002, como en 
la propia Ley Orgánica de Educación (LOE) española del año 2006. La «Declaración Universal de la UNESCO sobre la diversidad cultural», ya en 2001, señaló el papel de las lenguas en la construcción de una ciudadanía democrática (Beacco y Byram, 2003) y supuso el punto de partida para el desarrollo de estudios y proyectos orientados hacia una educación plurilingüe e intercultural (González Piñeiro, Guillén Díaz y Vez, 2010). Este aspecto esencial de la educación está presente en la legislación educativa tanto europea como española y americana (Roca, 2007 y Souto, 2009). Así, en la «Recomendación del Comité de ministros a los Estados miembros relativa a la educación para la ciudadanía democrática» se declara, entre otros objetivos:

que la educación para la ciudadanía democrática es un factor de cohesión social, de comprensión mutua, de diálogo intercultural e interreligioso, y de solidaridad, que contribuye a promover el principio de igualdad entre hombres y mujeres, y que favorece el establecimiento de relaciones armoniosas y pacíficas en los pueblos y entre ellos, así como la defensa y el desarrollo de la sociedad y la cultura democráticas

Por su parte, la Ley Orgánica de Educación (LOE) española del año 2006 se hizo eco de estos propósitos en el Título Primero al señalar cuáles son los fines del sistema educativo español, se señalan en el artículo 2, entre otros, los siguientes:

a. El pleno desarrollo de la personalidad y de las capacidades de los alumnos.

b. La educación en el respeto de los derechos y libertades fundamentales, en la igualdad de derechos y oportunidades entre hombres y mujeres y en la igualdad de trato y no discriminación de las personas con discapacidad.

c. La educación en el ejercicio de la tolerancia y de la libertad dentro de los principios democráticos de convivencia, así como en la prevención de conflictos y la resolución pacífica de los mismos.

d. La educación en la responsabilidad individual y en el mérito y esfuerzo personal.

e. La formación para la paz, el respeto a los derechos humanos, la vida en común, la cohesión social, la cooperación y solidaridad entre los pueblos así como la adquisición de valores que propicien el respeto hacia los seres vivos y el medio ambiente, en particular al valor de los espacios forestales y el desarrollo sostenible. 
Más recientemente, la Ley Orgánica para la Mejora de la Calidad Educativa LOMCE (2013) señala explícitamente como uno de los objetivos del sistema educativo español «la preparación para el ejercicio de la ciudadanía y para la participación activa en la vida económica, social y cultural con actitudes críticas». Se trata pues de poner en marcha un «conocimiento en acción» (Roca, 2007) capaz de convertirse en saberes productivos (La Porta, 2009) y constructivos que impliquen el crecimiento personal y el desarrollo justo de la comunidad.

Desde hace ya varias décadas, en España $-y$, en concreto en la Comunidad Valenciana-, en las aulas de secundaria conviven un gran número de adolescentes de distintas nacionalidades y lenguas (ver tabla 1), muchos de los cuales tienen que simultanear los procesos de aprendizaje de la lengua y el avance en el currículum escolar, con su propio proceso de integración social y cultural. Esto último supone para el estudiante el conocimiento y la asimilación de comportamientos y actitudes en muchos casos ajenos a los propios y que acrecientan mucho el sentimiento de extrañeza ya de por sí bastante evidente debido al desconocimiento del idioma.

\begin{tabular}{|c|c|c|c|c|c|c|}
\hline \multicolumn{7}{|c|}{ Alumnado extranjero } \\
\hline Curso & Alicante & Castellón & Valencia & C. Valenciana & España & $\% \mathrm{CV} / \mathrm{E}$ \\
\hline $2015-16$ & 39.596 & 14.392 & 37.068 & 91.056 & 716.736 & 12,7 \\
\hline $2016-17$ & 39.432 & 14.245 & 37.593 & 91.270 & 721.609 & 12,6 \\
\hline 2017-18 & 42.523 & 14.22 & 39.641 & 96.390 & 751.390 & 12,9 \\
\hline 2018-19 & 46.047 & 14.598 & 42.662 & 103.307 & 795.525 & 13,0 \\
\hline
\end{tabular}

Tabla 1. Conselleria de Educación, Cultura y Deporte. Estadística de enseñanzas no universitarias (centros, matrícula, graduados y personal). https://pegv.gva.es/es/temas/sociedad/educacion/estadisticadelasensenanzasnouniversitarias?viewUrl163713194=\%2Fauto\%2Fscpd\%2Fweb\%2F30501NoUniversitarias\%2Faecv00310_c.html

En nuestra opinión sería deseable, para facilitar este proceso complejo de conocimiento, no desaprovechar la ocasión de implementar las habilidades instrumentales - lingüísticas y sociales- de los estudiantes que no hablan español 
en ningún momento de su formación curricular. En ese sentido se posiciona para la enseñanza de segundas lenguas, el MCER que reitera, en el capítulo 2, el concepto de actividad en relación con la enseñanza-aprendizaje de lenguas:

El enfoque aquí adoptado, en sentido general, se centra en la acción en la medida en que considera a los usuarios y alumnos que aprenden una lengua principalmente como agentes sociales, es decir, como miembros de una sociedad que tiene tareas (no sólo relacionadas con la lengua) que llevar a cabo en una serie determinada de circunstancias, en un entorno específico y dentro de un campo de acción concreto.

Más recientemente, el informe de la Modern Language Association (MLA) sobre la enseñanza de segundas lenguas en la educación superior (2007) insiste en la idea de formar «educated speakers who have deep translingual and transcultural competence» (Barnes-Karol y Bornes, 2010). En este sentido, la introducción de competencias relacionadas con la interculturalidad o con la inclusión de dimensiones sociales y afectivas se orienta, precisamente, a inculcar en el estudiante valores de respeto y comprensión hacia la otredad. Se trata, por tanto, ahora también en L2/LE, de aproximarse al lenguaje atendiendo a su naturaleza de instrumento esencial para comprender el mundo: «referring to the manner in which language is a tool for articulating sense of relationship and of experience, and hence for learning about one's world» (Halliday apud Christie, F. \& Unsworth, L., 2006).

En el caso de español como lengua extranjera, las prácticas más cercanas a esta vertiente de la enseñanza de lenguas tienen que ver con la enseñanza de la interculturalidad y la necesidad de inculcar en el estudiante valores de respeto y comprensión hacia la otredad que suponen una lengua y una cultura nuevas (Marimón, 2016).

En este sentido, nos ha parecido que los textos de Educación para la Ciudadanía pueden ser un material idóneo para trabajar con los estudiantes no nativos los componentes de la competencia verbal mientras descubren y cuestionan los valores propios de una sociedad democrática en la que deberán actuar como agentes sociales y hablantes interculturales. No se trata de utilizar los textos del libro de EPC como un libro de lengua. Se trata de aprender a comprender al mismo tiempo el lenguaje y lo que nos trasmite y de saber interpretarlo en el futuro. 


\section{UN MODELO PARA INTEGRAR LENGUAJE Y CONOCIMIENTO DEL MUNDO}

Para llevar a cabo este trabajo hemos manejado tres libros de texto de la asignatura Educación para la Ciudadanía de tercer curso de la ESO (ver bibliografía) y, de ellos, el capítulo o apartado dedicado a los derechos de las mujeres.

El análisis concreto y dadas las limitaciones de este trabajo, se ha realizado sobre la primera página del apartado 3 titulado «La conquista de los derechos de las mujeres» incluido en capítulo 3: «Tengo derechos y deberes» del libro Educación para la Ciudadanía. ESO, editorial Edebe, pág. 44. Además, se aportarán fragmentos de los otros dos libros de texto con el fin de observar las diferencias en el planteamiento de unos y otros manuales.

La idea es aplicar de manera simplificada el modelo de Halliday que el PCIC utiliza para integrar al objeto y al sujeto del aprendizaje pero que no llega a desarrollar. Como es bien sabido, para la LSF (Eggins, 2002), el lenguaje, entendido como un sistema de opciones se organiza para realizar tres funciones o construir tres tipos distintos de significado: el ideacional o experiencial, el interpersonal y el textual. En el cuadro 1 se explicitan las tres metafunciones y las parcelas del significado a la que afectan cada una de ellas:

\section{METAFUNCIÓN IDEACIONAL EXPERIENCIAL quién hace qué a quién, afirmar, dar órdenes, qué ponemos primero, qué cuándo y dónde... METAFUNCIÓN METAFUNCIÓN \\ $O$ INTERPERSONAL TEXTUAL expresar seguridad... dejamos implicito...}

Cuadro 1. Elaboración propia a partir de Eggins, 2002.

Por su parte, estas tres metafunciones se realizan — se materializan- a través de los niveles lingüísticos y de las opciones expresivas que estos posibilitan. La perspectiva semiótica propone que el contenido de un enunciado depende de su semántica y de la estructura léxico-gramatical. Desde el nivel semántico se 
construye y observa el discurso como una unidad, de ahí que sean los recursos del nivel textual: cohesión léxica, estructura del intercambio o referencia los que sean tenidos en cuenta como generadores de significación. Desde el nivel léxico-gramatical, el discurso se observa como una organización sintáctica por lo que la unidad de análisis es la cláusula —la oración-. Transitividad, modalidad y tema son algunos de los mecanismos que realizan este nivel del significado. En el cuadro 2 siguiente vemos la relación entre los significados o funciones del lenguaje -ideacional, interpersonal y textual_- y los mecanismos semánticos y léxico-gramaticales que hacen posible y revelan al mismo tiempo esa significación:

\begin{tabular}{|c|c|c|}
\hline & SEMÁNTICA & LÉXICO-GRAMÁTICA \\
\hline $\begin{array}{lr}\text { El significado } \\
\text { ideacional se } \\
\text { expresa }\end{array}$ & $\begin{array}{l}\text { Cohesión léxica } \\
\text { Relaciones } \\
\text { conjuntivas }\end{array}$ & $\frac{\text { Transitividad }}{\text { Relaciones lógico-semánticas }}$ \\
\hline $\begin{array}{l}\text { El significado } \\
\text { interpersonal se } \\
\text { expresa }\end{array}$ & $\begin{array}{l}\text { Funciones del habla } \\
\text { Estructura del } \\
\text { intercambio }\end{array}$ & $\begin{array}{l}\text { Modo, modalidad } \\
\text { Actitud }\end{array}$ \\
\hline $\begin{array}{lr}\text { El significado } \\
\text { textual } & \text { se } \\
\text { expresa } & \end{array}$ & $\begin{array}{l}\text { Referencia } \\
\text { Conjunción }\end{array}$ & $\begin{array}{l}\text { Tema: estructura de la } \\
\text { información } \\
\text { Nominalizaciones }\end{array}$ \\
\hline
\end{tabular}

Cuadro 2. Elaboración propia a partir de Eggins, 2002.

A partir de este modelo, la LSF se propone analizar cómo el lenguaje crea significados - experienciales, interpersonales, textuales - mediante el uso de determinadas opciones semánticas y léxico-gramaticales. Esto permite no sólo analizar aspectos esenciales de la organización de la lengua (española), sino que, al concebir el lenguaje como un sistema de opciones que se actualiza en cada enunciado, para cada hablante, según qué necesidad, a tenor de qué contexto, podemos entender los textos como el resultado de la selección de un hablante que elige unos términos para significar y deja otros - conquista, historia - , que asume una actitud frente a su propio enunciado frente a otras posibilidades — debemos, se puede_-, que prefiere priorizar una determinada información y minimizar otra — se reclama, luchan-. 
Pero, además, entender el lenguaje, en cualquiera de sus manifestaciones, como el resultado de un proceso de conocimiento, de un acto de relación y de una voluntad de organización, tal y como lo plantea la LSF, sitúa el centro de atención ante cualquier enunciado sobre tres ejes fundamentales que son las dimensiones básicas sobre las que se sustenta cualquier actividad de aprendizaje y comprensión en L2: la realidad experiencial, el sujeto y el propio sistema de la lengua.

Las posibilidades didácticas de este planteamiento para la enseñanza del español como LE/L2 son muchas pero muy pocas, al menos en España, las propuestas en el que se haga mención de la práctica de significados ideativos, discursivos e interpersonales en el aula de ELE (Castañeda y Alonso Raya, 2009; Marimón, 2016)

\section{UN EJEMPLO DE PRÁCTICA EN EL AULA: LOS TEXTOS DE EDUCACIÓN PARA LA CIUDADANÍA}

El significado experiencial: Para trabajar sobre el significado experiencial se pueden realizar actividades dirigidas a llamar la atención sobre las relaciones léxicas cohesivas (Marimón, 2008). El primer paso puede ser la lectura del título: «La conquista de los derechos de las mujeres», que sitúa a los estudiantes ante tres conceptos que van a resultar esenciales y que señalarán enseguida: «conquista», «mujeres» $y$ «derechos». Es importante estar seguros de que conocen las nociones que se manejan en el tema pues conceptos como «derecho», «discriminación» o «igualdad» que aparecen con frecuencia pertenecen a los niveles B1-B2 del $P C I C$. Sería muy recomendable, por tanto, una actividad de pre-lectura del tipo $\mathrm{V} / \mathrm{F}$, unir con flechas o asociar imágenes puede servir para activarles o darles a conocer estos conceptos en español.

Tras la lectura del texto — solos o en parejas-, se puede abordar ya un primer acceso consciente al texto. Para ello, se les propone una actividad sencilla y lúdica: intentar adivinar cuántas veces se repiten en el texto las palabras «mujer» o «mujeres». Tras sus «apuestas» les indicamos que subrayen todas las ocurrencias y las cuenten. Verán que son un total de 18 - suelen decir muchas menos lo cual es lógico pues los hablantes no suelen ser conscientes de la reiteración discursiva que facilita la comprensión-. Les llamamos entonces la atención 


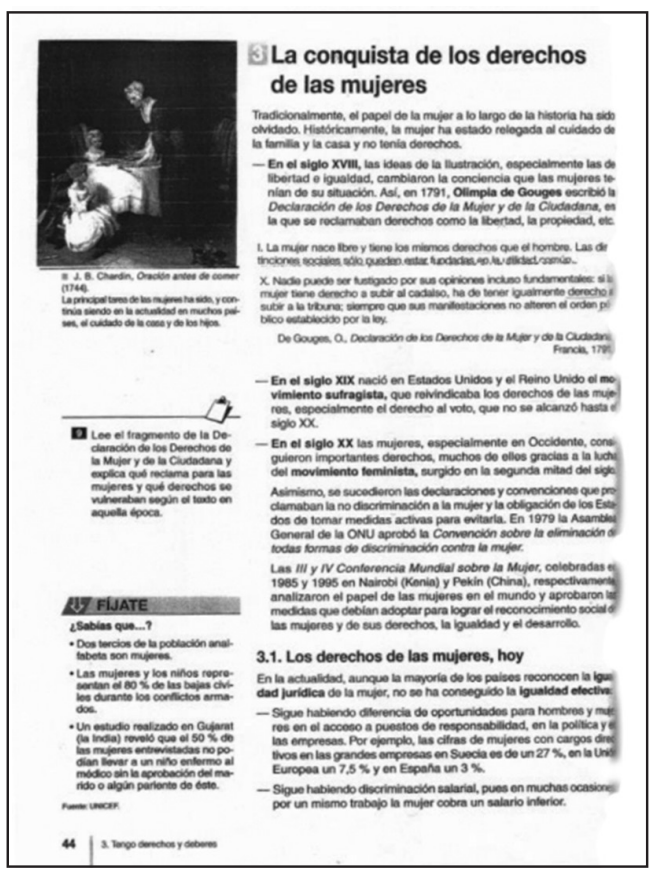

Texto 1. Educación para la Ciudadanía. ESO (2007). Madrid: Santillana

sobre el hecho de que, cuando se trata un tema, la palabra clave suele repetirse muchas veces. Buscamos ahora otra palabra clave, «derecho». Los alumnos comprobarán entonces que derecho aparece 12 veces, pero les llamaremos la atención sobre otras palabras con las que esta está relacionada: «igualdad», que aparece 4 veces, «libertad», tres y «no-discriminación», 1. Todas ellas forman parte del concepto más general «derecho» que es su hiperónimo. Un sencillo mapa conceptual ayudará a visualizarlo.

Quizá alguien se haya dado cuenta de que, junto a no-discriminación aparece «discriminación» que es lo contrario. Busquemos ahora, pues, antónimos y encontraremos: «diferencia», «distinción social», «relegada».

Según el título, los derechos que acabamos de nombrar son una «conquista», eso significa que no es algo que haya sido así siempre, de hecho, "conquistar» significa según el diccionario de la RAE: «Ganar, conseguir algo, generalmente 
con esfuerzo, habilidad o venciendo algunas dificultades». Aplicado a nuestro texto, significa que alguien -las mujeres - estaban en una situación anterior de no-derechos y que han tenido que esforzarse y vencer dificultades para encontrarse ahora en una situación de «derechos», pero ¿quién se ha esforzado? ¿Quién ha puesto dificultades? ¿qué han conseguido? ¿cómo lo han hecho? Un análisis del nivel léxico-gramatical nos va a ayudar a repartir papeles activos entre los participantes en esta «conquista».

Los actores discursivos: Para ello les vamos a pedir a los estudiantes que, por parejas, escriban en su cuaderno todas las oraciones del texto y vamos a señalar con ellos los verbos (las acciones) y quienes las hacen y reciben (actores). Si no hay sujeto, deberán también indicarlo.

Así, por ejemplo, encontramos:

«El papel de la mujer a lo largo de la historia ha sido olvidado» "La mujer ha estado relegada al cuidado de la familia y la casa y no tenía derechos» «Las ideas de la llustración cambiaron la conciencia que las mujeres tenían de su situación»

«Olimpia de Gouges escribió la Declaración de los Derechos de la Mujer y de la Ciudadana en la que se reclamaban derechos como la libertad, la propiedad, etc.» «En el s. XX nació en EEUU y el Reino Unido el movimiento sufragista, que reivindicaba los derechos de las mujeres, especialmente el derecho al voto que no se alcanzó hasta el siglo $X X »$

«En el siglo XX, especialmente en Occidente, las mujeres consiguieron importantes derechos, muchos de ellos gracias a la lucha del movimiento feminista» «Se sucedieron las declaraciones y convenciones que proclamaban la no discriminación de la mujer"

«La Asamblea general de la ONU aprobó»

«Las III y IV Conferencia Mundial sobre la Mujer [...] analizaron [...] y aprobaron medida que debían adoptar para lograr el reconocimiento social de las mujeres»

«En la actualidad no se ha conseguido la igualdad efectiva» «sigue habiendo diferencia de oportunidades para hombres y mujeres en el acceso a puestos de responsabilidad» 
«las cifras de mujeres con cargos directivos [...] es»

«sigue habiendo discriminación salarial»

«por un mismo trabajo la mujer cobra un salario inferior»

Luego les diremos que señalen otra vez la palabra mujer, los juntaremos en pequeños grupos y les propondremos que se fijen en el lugar que ocupa la palabra «mujer» dentro de las oraciones: ¿ocupa un lugar principal o son siempre lugares de «dependencia», de "subordinación»? A partir de este análisis se les proponen las siguientes preguntas para reflexionar y comentar: ¿cuántas veces las mujeres aparecen como sujetos, haciendo algo? ¿se le asocian actividades positivas? ¿Qué otras personas hacen cosas? ¿o no se nombra a muchas personas? En el caso de que se encuentren otros actores, se pide a cada grupo que realice una lista. Se verá sin embargo que domina la impersonalidad y la falta de asignación de acciones. Esta indeterminación, unida a la escasa presencia de las mujeres en papel actancial activa nos lleva a realizar la siguiente pregunta: Según este texto ¿ha sido la mujer protagonista de su propia historia?

Con el fin de mostrar con más claridad la ausencia de agentividad y la pasivización del elemento femenino que revela el texto analizado, se realizará una comparación entre este y fragmentos pertenecientes a otros textos de la misma materia, más breves, pero muy elocuentes.

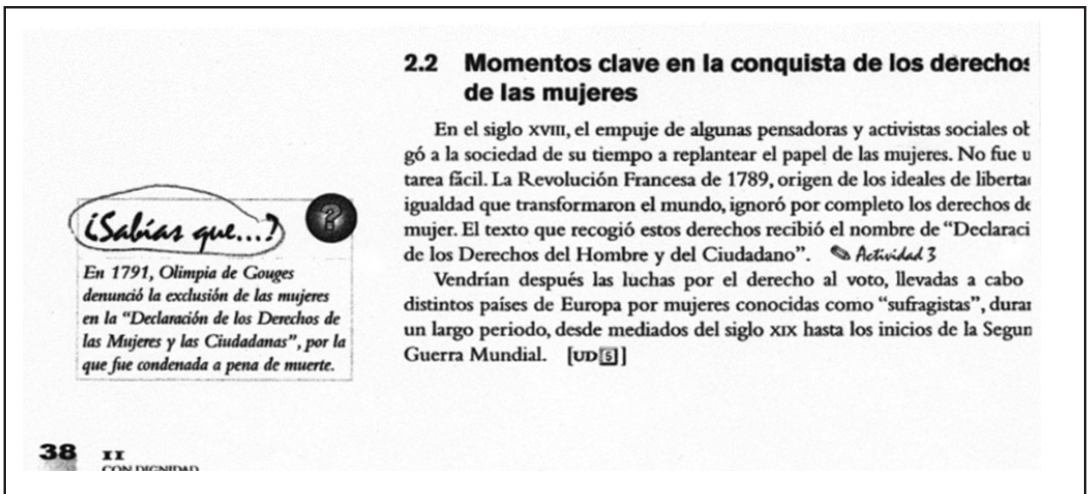

Texto 2. Educación para la Ciudadanía. ESO (2007). Madrid: Santillana 
Se vuelve a unir a los estudiantes por parejas y se les proponen las siguientes actividades:

Lee ahora este pequeño texto, fíjate en las palabras y señala ¿quién aparece aquí que apenas es nombrado en el texto 1 ? iel hombre, claro! ¿con qué palabras aparece nombrado? ¿y las mujeres? Antes hemos hablado de la antítesis, de palabras que significan lo contrario ¿podrías señalar qué actividades se asocian a hombres y mujeres?

\section{La mujer en las sociedades antiguas}

En las sociedades antiguas, el papel de la mujer era de subordinación respecto al hombre. En el mundo greco-romano, las leyes permitían al marido el divorcio y el repudio de la esposa sin que tuviese que alegar ningún motivo especial, pero la esposa no tenía derecho a hacer lo mismo.

Las jóvenes se casaban muy pronto, a los quince años o antes, con quien decidía su padre, y permanecían dedicadas al cuidado de su hogar, de los hijos y de los esclavos sin que se les permitirera praticipra ers ${ }^{1} a$ ' vida pública, 'y apetras tetíratı acteso a la educación.

Texto 3. Educación para la Ciudadanía. ESO (2008). Madrid: Anaya entre culturas.

Lee ahora el texto 3 que también trata de la conquista de los derechos. Busca palabras relacionadas con este concepto ¿a quién se asocian?

A estas alturas quizá el estudiante se ha dado cuenta ya de que al primer texto le faltan actores — no sabemos quién hace las cosas-, ni hay demasiada precisión ni variación en los conceptos ¿a qué derechos se refiere?

Te propongo algo: ahora que ya sabes más del tema, reescribe las frases que consideres del primer texto, reparte bien los papeles, nombra las acciones y los actores.

Si piensas que ahora el lenguaje expresa mejor el significado del texto, cambia también el título: las mujeres conquistan sus derechos a lo largo de la historia ¿por qué no? 


\section{CONCLUSIONES}

A comienzos de los años 80 , Halliday y sus colaboradores elaboraron un Proyecto educativo para la escuela australiana (Ghio, 2008) en el que se proponía el estudio de la lengua desde tres perspectivas: aprender la lengua —-dominio básico de leer, escribir, comprender y hablar-, aprender a través de la lengua — «tomar conciencia de las diversas maneras de expresar la experiencia»- y aprender acerca de la lengua — su sistema gramatical, su estatus como institución, sus variedades-. Se trataba de integrar destrezas comunicativas y conocimiento declarativo de naturaleza lingüística en un sistema escolar poco habituado a que los estudiantes tuvieran que estudiar el sistema gramatical del inglés. El proyecto estaba pensado para hablantes nativos y fue reformulado en varias ocasiones (Martin, por ejemplo, concibió un modelo de enseñanza de la lengua basado en los géneros discursivos). Independientemente de su vigencia en Australia, el planteamiento tuvo repercusión en todos los sistemas educativos Occidentales pues encajaba perfectamente con los modelos activos y comunicativos que las diferentes reformas estaban poniendo en práctica. Así, por el ejemplo en la Introducción de la LOGSE se dice que «la educación lingüística y literaria estará guiada por la consideración del lenguaje como actividad que tiene como funciones básicas la comunicación y la representación de la realidad física y social»:

Aplicado al aprendizaje de segundas lenguas esta propuesta se integra plenamente en el enfoque comunicativo. Así, aprender la lengua está estrechamente relacionado con la adquisición de las cuatro destrezas básicas - comprensión/ expresión oral y escrita-, mientras que aprender acerca de la lengua significa introducir conocimientos sobre el sistema lingüístico en sus distintos niveles -la gramática, el léxico, la fonética - y las posibilidades expresivas de un idioma -los registros, las variedades-. Pero el concepto sobre el que quería llamar la atención es, precisamente, el de aprender a través de la lengua. De lo que se trata en este caso es de hacer al alumno consciente de que la lengua no es un mero instrumento de transmisión a través del cual «pasa» el conocimiento y por eso debemos dominarla. Más allá de eso, lo esencial es hacer ver al estudiante que la lengua «es» conocimiento, que ser consciente, por ejemplo, de la forma de organización de un enunciado - elegir explicar, narrar o describir algo-; de la selección del vocabulario -nombrar o evitar nombrar algo o a alguien, como hemos visto; del 
reparto de papeles — quién hace o quien no hace las acciones que se enuncian-; de lo que se pone en primer lugar y de lo que sale siempre al final, es el resultado de elecciones más o menos conscientes por parte de alguien que, necesariamente, nos hacen ver la realidad desde un punto de vista. Y saber eso y saber, además, que se pueden cambiar los papeles - y hacerlo- o nombrar lo no nombrado o hacer actuar a quien no actúa —y hacerlo también- sitúa al estudiante en un papel verdaderamente consciente y activo no sólo frente a la nueva lengua, sino frente la nueva realidad social que se abre ante él. Al aprender la lengua y acerca de ella, el estudiante se desarrollará como un agente social conocedor del sistema y de su uso. Al aprender a través de la lengua, el estudiante podrá, además, acercarse a las distintas posibilidades de pensar el mundo a través del lenguaje y de pensarse a sí mismo y adquirir, siguiendo el PCC y como, en realidad, exige la sociedad actual, verdadera competencia como hablante intercultural.

\section{BIBLIOGRAFÍA}

Atienza Cerezo, E. (2007). Discurso e ideología en los textos de ciencias sociales. Discurso y Sociedad 1, (04), 543-573

Barnes-Karol G. y M.A. Broner (2010). Using Images as Springboards to Teach Cultural Perspectives in the Light of the Ideals of the MLA Report. Foreing Language Annals 43 (3), 422-445.

Beacco, J.C. y Michael B. (2003). Guide for the development of language education policies in Europe. From linguistic diversity to plurilingual education. Language Policy Division, Council of Europe: Strasbourg.

Castañeda Castro, A. y Alonso Raya, R. (2009). La percepción de la gramática. Aportaciones de la lingüística cognitiva y la pragmática a la enseñanza del español/ LE. MarCoELE 8, 1-33.

Cenoz, J. (2004) El concepto de competencia comunicativa. En Sánchez Lobato, J. y Santos Gargallo, I. Vademécum para la formación de profesores. Enseñar español como segunda lengua/lengua extranjera. Madrid: SGEL, 449-465. 
Christie, F. y L. Unsworth (2006). «Developing dimensions of an educational linguistics». En J. Webster, C. Matthiessen, y R. Hasan (Eds.), Continuing Discourse on Language: A Functional Perspective, (pp. 225-258). London: Equinox.

Coicaud, S. (2009). Jóvenes, escuela media y enseñanza innovadora de la ciudadanía democrática. III Congreso internacional de educación. Construcciones y perspectivas, Santa Fe: UNL, 1-8.

Eggins, S. (2002). Introducción a la lingüística sistémica. Logroño: Universidad de la Rioja. Gabalachis, G. (2009). «Construyendo un modelo educativo generador de ciudadanía e inclusión social», III Congreso internacional de educación. Construcciones y perspectivas, Santa Fe: UNL, 1-8.

Ghio, E. y Fernández, M.D. (2008). Lingüística sistémico funcional aplicada a la lengua española, Santa Fe: Universidad Nacional del Litoral

González Piñeiro, M.C. Guillén y J.M. Vez (2010). Didáctica de las lenguas modernas. Competencia plurilingüe e intercultural. Madrid: Síntesis.

Halliday, M.A.K. (1975). Estructura y función del lenguaje. En Lyons, J.(ed.). Nuevos horizontes de la lingüística. Madrid: Alianza Editorial, 54-173.

Halliday, M.A.K. (1982). El lenguaje como semiótica social: la interpretación social del lenguaje y del significado, México: Fondo de Cultura Económica.

https://www.boe.es/buscar/pdf/2013/BOE-A-2013-12886-consolidado.pdf

La Porta, P.A. (2009). Educación para la participación como requisito para la ciudadanía plena. III Congreso internacional de educación. Construcciones y perspectivas. Santa Fe: UNL, 1-8.

Ley Orgánica De Educación (LOE) (2006). http://www.boe.es/boe/dias/2006/05/04/ pdfs/A17158-17207.pdf

Ley Orgánica para la mejora de la calidad educativa (LOMCE) (2013)

Marimón Llorca, C. (2008). Análisis de textos en español. Teoría y práctica. Alicante: Universidad de Alicante.

Marimón Llorca, C. (2009). Estrategias discursivas para comprender: enseñar educación para la ciudadanía a estudiantes no nativos. En A. Vera Lujan e I. Martínez Santander (Eds.), XX Congreso Internacional de la ASELE. El español en contextos específicos: enseñanza e investigación. Comillas 2009. Pamplona: ASELE, Fundación Comillas, pp. 697-717. 
Marimón Llorca, C. (2016). Hacia una dimensión crítica en la enseñanza de español como lengua extranjera: la Competencia Comunicativa Intercultural Crítica (CCIC). RESLA, 29 (1), p. 191-2011.

Plan Curricular del Instituto Cervantes (2007). Edelsa: Instituto Cervantes.

Recomendación (2002)12 del Comité de Ministros a los Estados miembros relativa a la educación para la ciudadanía democrática http://www.coe.int/t/dg4/education/ edc/Source/Pdf/Documents/By_Country/Spain/2002_38_Rec2002_12_Es.PDF

Roca Cobo, E. (2007). La educación para la ciudadanía y los derechos humanos en la LOE y en sus desarrollos. En Educación para la ciudadanía, Madrid: Instituto de la Mujer, 19-36.

Souto Galván, B. (2009). Educación para la ciudadanía: un estudio comparado entre los ordenamientos español y portugués. III Congreso internacional de educación. Construcciones y perspectiva. Santa Fe: UNL, 1-8.

\section{TEXTOS UTILIZADOS}

Texto 1. Educación para la Ciudadanía. ESO (2008): Madrid, Edebe.

Texto 2. Educación para la Ciudadanía. ESO (2007): Madrid, Santillana.

Texto 3. Educación para la Ciudadanía. ESO (2008): Madrid, Anaya entre culturas. 